\title{
Estudo Prospectivo da Soldagem Dissimilar pelo Processo FSW de Ligas de Alumínio AA 7075 e AA 5083
}

\section{Prospective Study of Dissimilar Welding by FSW Process of the Aluminum Alloys AA 7075 and AA 5083}

Déborah Brito Colaço ${ }^{1}$, Mattheus Apolinário Ribeiro', Amós Freitas de Figueiredo², Oclávio Coutinho dos Santos², Emanuel Luciano Lunes Medeiros ${ }^{2}$, Theophilo Moura Maciel ${ }^{1}$, Raphael Henrique Falcão de Melo ${ }^{2 \star}$

\section{RESUMO}

Uma relação de boa resistência mecânica e baixo peso específico é uma solicitação premente do setor de transporte, seja aéreo, aquaviário ou terrestre. Neste sentido as ligas de alumínio surgem como solução de engenharia, pois além de apresentar as características necessárias possuem boa resistência à oxidação e alta reciclabilidade. A união de ligas de alumínio dissimilares pode ser utilizada nos mais variados cenários a fim de aproveitar as melhores propriedades de cada um dos materiais a unir, entretanto, a utilização de soldagens convencionais por fusão não se torna apropriada devido a grande diferença de propriedades físicas e químicas entre os componentes a serem unidos. A soldagem por fricção e mistura mecânica Friction Stir Welding (FSW) torna-se uma alternativa viável para a realização de soldagens dissimilares, já que é realizada no estado sólido, evitando as desvantagens da soldagem por fusão. Este trabalho teve por objetivo caracterizar e avaliar os níveis de tensões residuais de juntas de ligas de alumínio dissimilares 5083 e 7075 soldadas pelo processo FSW utilizando diferentes parâmetros de soldagem. Para tanto, nove juntas soldadas foram confeccionadas. Ensaios mecânicos de dobramento de raiz a $60^{\circ}$, tração uniaxial, determinação do perfil de dureza e de tensões residuais das juntas soldadas foram realizados para caracterização e análise das propriedades. Foi observado que valores de avanço de 180 mm/min, rotação de 345 RPM sem offset forneceram melhores propriedades mecânicas, além de um menor nível de tensões residuais trativas.

Palavras-chave: Soldagem dissimilar, Friction Stir Welding, Liga de alumínio.

\section{ABSTRACT}

A relationship of good mechanical resistance and low specific gravity is an urgent request of the transport sector, be it aerial, waterway or terrestrial. In this way aluminum alloys appear as an engineering solution, as well as having the necessary characteristics it has good resistance to oxidation and high recyclability. The combination of dissimilar aluminum alloys can be used in a variety of scenarios in order to take advantage of the best properties of each of the materials to be joined, however, the use of conventional fusion welding is not appropriate due to the large difference in physical and chemical properties between the components to be joined. Friction welding and mechanical mixing (FSW) becomes a viable alternative for the realization of dissimilar welding, since it is carried out in the solid state, avoiding the disadvantages of fusion welding. This work aims to characterize and evaluate the residual stress levels of dissimilar aluminum alloy joints 5083 and 7075 welded by the FSW process using different welding parameters. For that, nine welded joints were made. Mechanical tests of root folding at $60^{\circ}$, uniaxia traction, determination of the hardness profile and residual stresses of welded joints were carried out to characterize and analyze the properties. It was observed that advance values of $180 \mathrm{~mm} / \mathrm{min}$, rotation of 345 RPM without offset provided better mechanical properties, besides a lower level of residual tensile stresses.

Keywords: Dissimilar welding, Friction Stir Welding, Aluminum alloy.

1. Universidade Federal de Campina Grande - Departamento de Engenharia Mecânica - Campina Grande (PB), Brasil 2.Instituto Federal de Educação, Ciência e Tecnologia da Paraíba - Unidade Acadêmica de Indústria - Campina Grande (PB), Brasil Autor correspondente: raphael.melo@ifpb.edu.br Recebido: 10 Mar 2019 Aprovado: 31 Jul 2019 


\section{INTRODUÇÃO}

A união de diferentes materiais é muito atraente para a indústria, já que reduz o uso de materiais de maior custo, utilizando-os somente quando necessário. A união dissimilar pode ser frequentemente utilizada em muitos cenários, principalmente nas indústrias automotivas, aeroespaciais e de construção naval ${ }^{1}$. Entretanto, a utilização da soldagem convencional por fusão não é apropriada para realizar a união de materiais dissimilares, devido a grande diferença de propriedades físicas e químicas entre os componentes a serem unidos ${ }^{2,3}$, causando diversos problemas, entre os quais se pode citar: porosidade, trincas de solidificação e reações químicas.

A soldagem por fricção e mistura mecânica FSW (Friction Stir Welding) torna-se uma alternativa para a realização de soldagens dissimilares, já que ela é realizada no estado sólido, evitando as desvantagens da soldagem por fusão ${ }^{1}$. Esse processo de soldagem é interessante tanto para as séries de ligas de alumínio termicamente tratáveis, que têm sua resistência mecânica aumentada pela formação de precipitados de fases, e baixa soldabilidade por processos de soldagem por fusão; como ligas da série $2 \mathrm{XXX}$ e $7 X X X^{4,5}$, como para as séries de ligas trabalháveis onde o aumento de resistência mecânica é obtido pela deformação mecânica a frio, tais como as ligas 1XXX, 3XXX, 4XXX e 5XXX; em função de benefícios como aumento da resistência à fadiga e menor nível de tensão residual ${ }^{6-8}$.

A técnica de soldagem (FSW) foi criada na Inglaterra em 1991, pelo The Welding Institute (TWI). O processo FSW ocorre no estado sólido, gerando calor por fricção e uma deformação plástica, provenientes do contato entre uma ferramenta não consumível e a interface da peça a ser soldada. Esse processo demonstra alta aptidão para soldar juntas de ligas de alumínio dissimilares, sendo os defeitos relacionados à presença de fases frágeis interdendríticas e eutéticas eliminados ${ }^{9}$. As dificuldades na soldagem por fusão de ligas de alumínio da série 7XXX devem-se à presença de inclusão de óxido, à formação de porosidade e às alterações na microestrutura. Tais dificuldades foram superadas pelo processo de soldagem FSW, que produz juntas soldadas com melhores propriedades mecânicas que àquelas fabricadas por processos de soldagem por fusão, a exemplo do Gas Tungsten Arc Welding (GTAW) e Gas Metal Arc Welding (GMAW) O processo FSW não utiliza gases de proteção, fluxos ou material de adição ${ }^{9,10}$.

Apesar das excelentes características do processo FSW, a soldagem de materiais dissimilares induz ao aparecimento de tensões residuais consideráveis devido à diferença entre os coeficientes de dilatação e contração térmica ${ }^{11}$. É de entendimento comum que as tensões residuais de soldagem atreladas à ocorrência de defeitos de soldagem possuem influência notável na vida em fadiga do material, no surgimento de trincas que acentuam fraturas, no processo de corrosão, entre outros ${ }^{12}$. O controle dos parâmetros de soldagem, além da verificação e aferição das tensões residuais é essencial para garantir um bom desempenho de juntas soldadas.
Neste contexto, esse prospectivo em juntas dissimilares de ligas de alumínio é um tema atual e relevante para a indústria nacional. Embora o processo FSW tenha merecido destaque, sendo objeto de estudo em diversos centros de pesquisa tecnológica no exterior, pouco se tem observado quanto a trabalhos realizados inteiramente no Brasil, especialmente no que se refere à soldagem dissimilar de ligas de alumínio pelo processo FSW ${ }^{13,14}$. Além disto, há carência na literatura técnica nacional e internacional a respeito da correlação entre tensões residuais, características mecânicas e metalúrgicas.

Assim, este trabalho tem por objetivo caracterizar e avaliar os níveis de tensões residuais de juntas de ligas de alumínio dissimilares AA 5083 e AA 7075 soldadas pelo processo FSW utilizando diferentes parâmetros de soldagem.

\section{MATERIAIS E MÉTODOS}

Foram utilizadas chapas de alumínio da liga 5083-O e 7075T651 com 4,76 mm de espessura. A designação $O$ representa o tratamento térmico de recozimento e a designação T651 representa o tratamento térmico de envelhecimento artificial. As composições químicas das chapas segundo o fabricante são apresentadas na Tabela 1 .

Tabela 1: Composição das ligas em estudo (\% em peso).

\begin{tabular}{|c|c|c|c|c|c|c|c|}
\hline & Mg & Fe & Cu & Mn & Cr & Zn & Al \\
\hline 5083 & 4.670 & 0.240 & 0.011 & 0.880 & 0.083 & 0.016 & Balanço \\
\hline 7075 & 2.632 & 0.114 & 1.623 & 0.060 & 0.192 & 5.498 & Balanço \\
\hline
\end{tabular}

A fim de averiguar a influência de diferentes parâmetros de soldagem na resistência mecânica e na magnitude das tensões residuais, foram realizadas nove soldagens pelo processo FSW variando os parâmetros de velocidade de avanço, velocidade de rotação e offset, como pode ser observado na Tabela 2. Foram estabelecidos como parâmetros fixos o ângulo de inclinação da ferramenta de $3^{\circ}$, a liga 5083-O no lado de avanço e a 7075-T651 no lado de recuo.

Tabela 2: Parâmetros de soldagem dissimilares pelo processo FSW.

\begin{tabular}{|c|c|c|c|}
\hline $\begin{array}{c}\text { Junta } \\
\text { soldada }\end{array}$ & $\begin{array}{c}\text { Velocidade de } \\
\text { avanço (mm/min) }\end{array}$ & $\begin{array}{c}\text { Velocidade de } \\
\text { rotação (RPM) }\end{array}$ & $\begin{array}{c}\text { Offset } \\
(\mathbf{m m})\end{array}$ \\
\hline 1 & 35 & 345 & 0 \\
\hline 2 & 180 & 345 & 0 \\
\hline 3 & 35 & 865 & 0 \\
\hline 4 & 180 & 865 & 0 \\
\hline 5 & 35 & 345 & 1 \\
\hline 6 & 180 & 345 & 1 \\
\hline 7 & 35 & 865 & 1 \\
\hline 8 & 180 & 865 & 1 \\
\hline 9 & 118 & 642 & 0,5 \\
\hline
\end{tabular}


As juntas soldadas foram confeccionadas utilizando uma fresadora universal automática modelo FU-300 da Diplomat. Na Fig. 1, apresentam-se as dimensões da junta soldada.

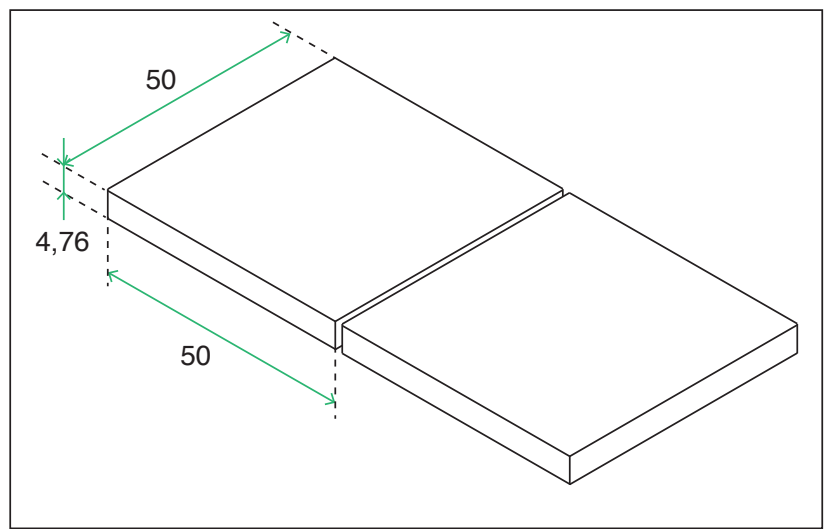

Figura 1: Esquema da junta de topo empregada neste trabalho (dimensões em milímetros).

Os corpos de prova para os ensaios de tração e dobramento a $60^{\circ}$ são apresentados nas Figs. 2 e 3, respectivamente e foram obtidos transversalmente ao cordão de solda. Esses ensaios foram realizados em uma máquina universal da Instron, modelo 5582. Nos ensaios de tração foram utilizados três corpos de prova para cada condição soldada, seguindo a norma ASTM E8/E8M - 16A, sendo aplicada uma taxa de deslocamento de $1 \mathrm{~mm} / \mathrm{min}$ até a ruptura dos corpos de prova. Já nos ensaios de dobramento foram utilizados dois corpos de prova para cada perfil de ferramenta, seguindo a norma ASTM E190-92, sendo os testes realizados voltados para raiz da junta.

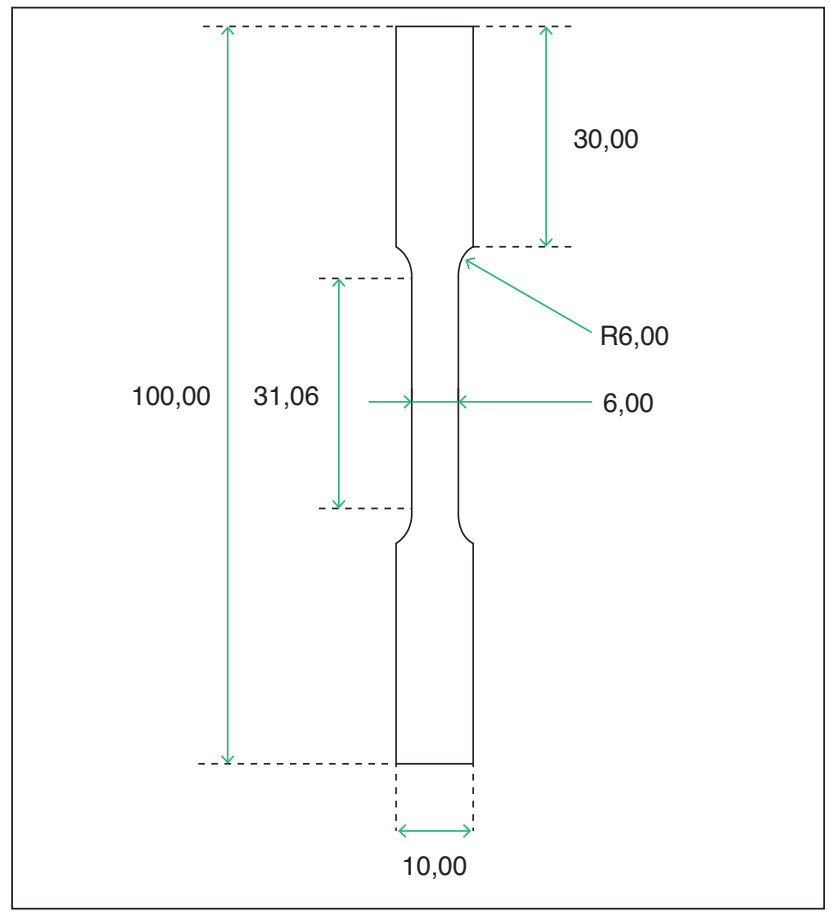

Figura 2: Corpo de prova de tração utilizado (dimensões em mm).

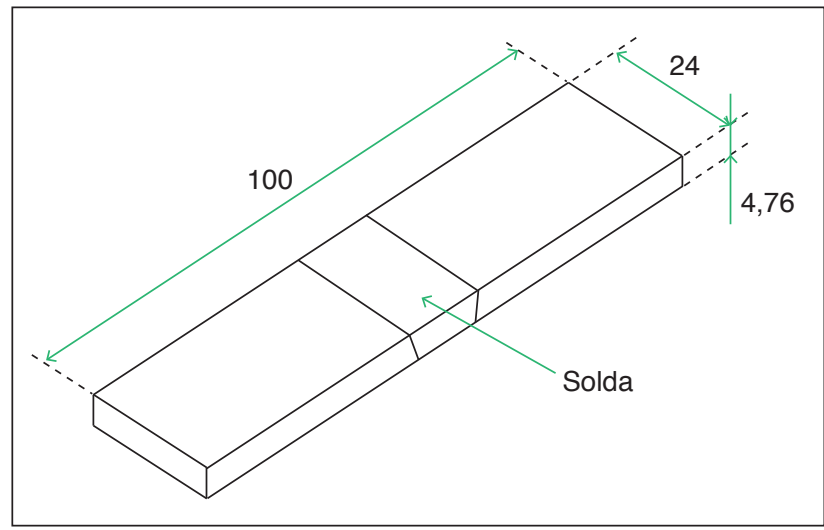

Figura 3: Corpo de prova de dobramento utilizado (dimensões em milímetros).

Os ensaios de dureza utilizando um microindentador Vickers (HV) foram executados aplicando-se uma carga de 50 gramasforça (gf), por 15 segundos, com espaçamento entre as impressões de $200 \mu \mathrm{m}$, conforme Fig. 4. Em cada amostra foram realizadas em torno de 150 medidas ao longo de apenas uma linha horizontal transversal ao cordão de solda utilizando um microdurômetro digital FM-700 da FutureTech.

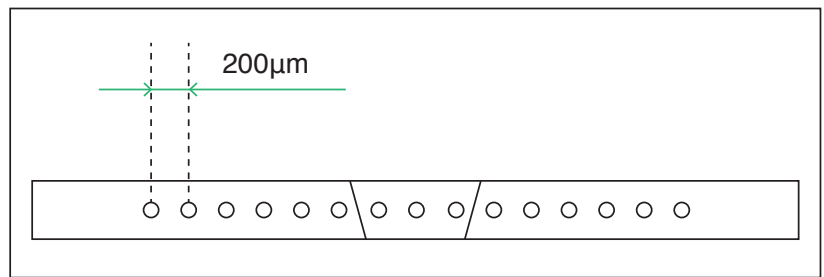

Figura 4: Posição onde foram realizadas as impressões de dureza.

Por fim, para determinar a magnitude das tensões residuais nas juntas soldadas, empregou-se uma variação da técnica semi-destrutiva do furo cego, conforme desenvolvido por Siqueira Filho et al. ${ }^{15}$. O método consiste em utilizar uma máquina de medição de coordenadas e determinar a variação das coordenadas pré-determinadas para o centro de um furo após a realização deste. Espera-se que ocorra variação das coordenadas em função da relaxação das tensões residuais de soldagem. Para tanto, são empregadas as Eqs. 1 e 2:

$$
\begin{aligned}
& \sigma_{x}=\frac{E}{1-v^{2}}\left(\varepsilon_{x}+v \varepsilon_{y}\right) \\
& \sigma_{y}=\frac{E}{1-v^{2}}\left(\varepsilon_{y}+v \varepsilon_{x}\right)
\end{aligned}
$$

onde $\sigma_{x}$ é a magnitude da tensão residual longitudinal; $\sigma_{y}$ é a magnitude da tensão residual transversal; $E$ é o módulo de elasticidade; $v$ é o coeficiente de Poisson; $\varepsilon_{x}$ é a deformação característica na direção longitudinal; $\varepsilon_{y}$ é a deformação característica na direção transversal. 
As coordenadas de referência foram determinadas em um programa CNC, em código FANUC, e os furos foram realizados utilizando-se um centro de usinagem D-600 da Romi. O esquema da furação é apresentado na Fig. 5. As coordenadas após a realização da furação foram determinadas utilizando uma Máquina de Medição de Coordenadas modelo TESAMICROHITE 3D, com resolução de $0,1 \mu \mathrm{m}$.

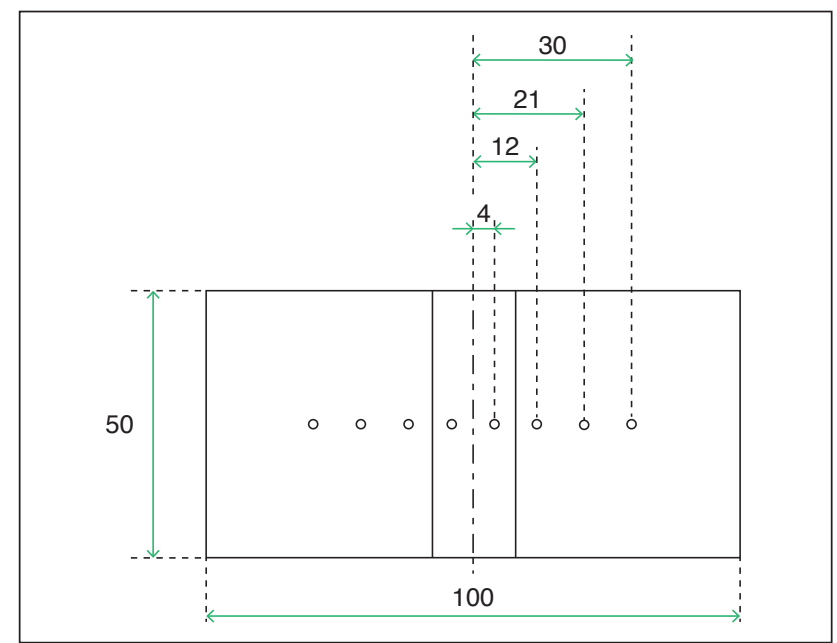

Figura 5: Esquema da furação para aferição da tensão residual (dimensões em milímetros).

\section{RESULTADOS E DISCUSSÃO}

Foram realizadas inspeções visuais superficiais em todas as nove juntas soldadas pelos diferentes parâmetros de soldagem, a fim de visualizar defeitos superficiais na solda, como falta de penetração, irregularidades na superfície da solda, espaços vazios ou túneis e excesso de rebarba (flash), já que esses defeitos podem influenciar nos resultados dos ensaios mecânicos posteriores. As juntas soldadas ficaram livres de defeitos em nível macro, como pode ser observado na Fig. 6, correspondente à junta do parâmetro 3 .

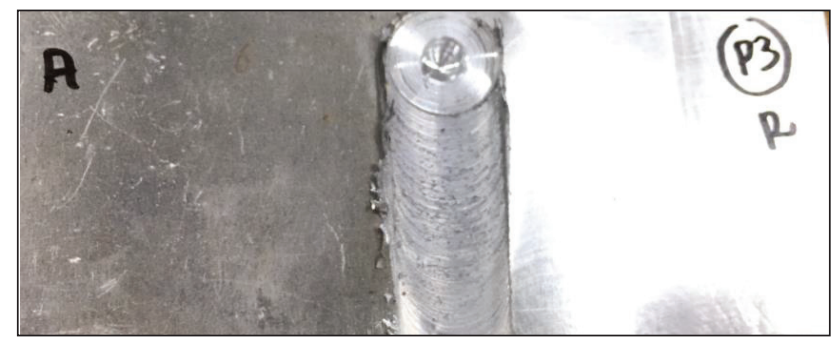

Figura 6: Junta soldada livre de defeitos.

A Fig. 7 ilustra os perfis de tensões residuais longitudinais e transversais. Quando são realizadas soldagens por processos convencionais de fusão, surgem nas regiões da zona termicamente afetada, tensões residuais trativas e na zona fundida, tensões residuais compressivas, já que o aquecimento excessivo nas regiões adjacentes tende a gerar tensões térmicas que, por seguinte, gera as tensões residuais.
Richards et al. ${ }^{16}$ e Altenkirch et al. ${ }^{17}$ investigaram as tensões residuais em soldas de liga de alumínio de alta resistência pelo processo por fricção. Eles encontraram uma distribuição de tensão longitudinal em forma de ' $M$ ', onde as tensões residuais de pico de tração estão localizadas, perto do limite da zona de ZTA. O perfil de tensão residual na forma de $\mathrm{M}$ foi atribuído por Richards et al. ${ }^{16}$ à natureza difusa do campo térmico visto em soldagens FSW em relação a outros processos de soldagem, combinado com o alto nível de amolecimento que ocorre com ligas de alumínio de alta resistência a temperaturas elevadas. Para os casos estudados neste trabalho não foram visualizadas distribuições de tensões residuais na forma $M$, devido às variações nos parâmetros de soldagem, que afeta a entrada de energia e os picos de temperaturas máximas atingidas, conforme apresentado na Fig.7.

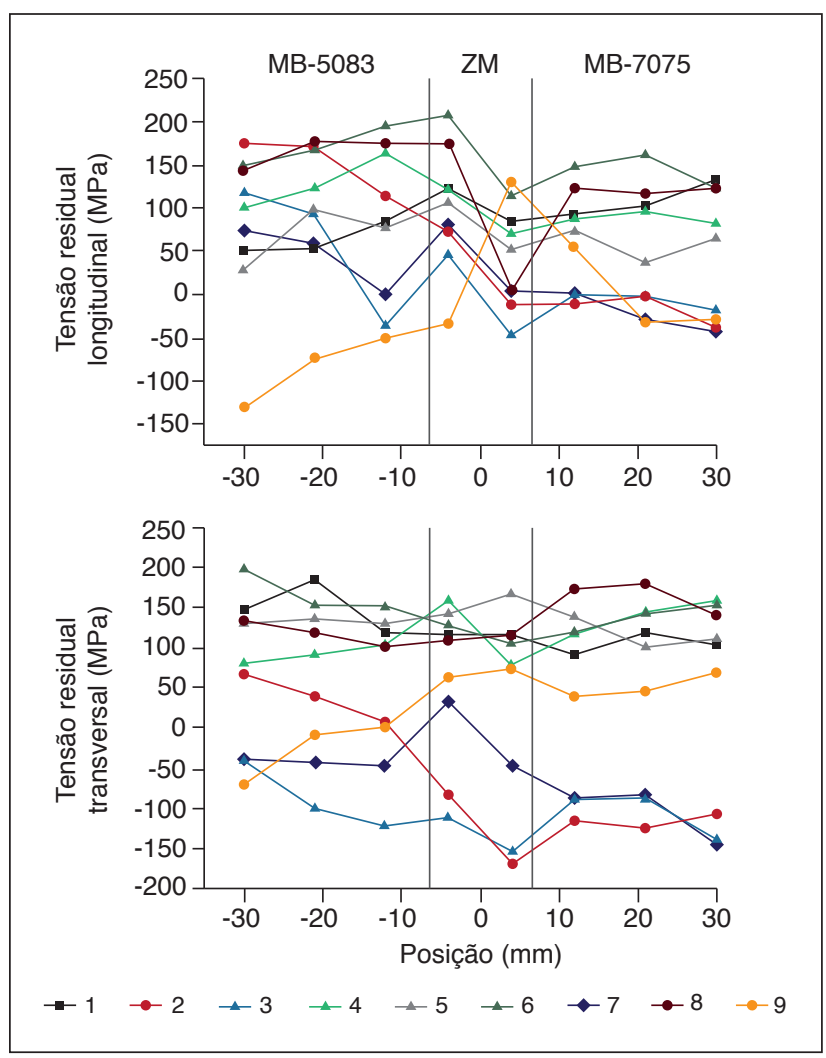

Figura 7: Magnitude das tensões residuais longitudinais e transversais nas juntas soldadas.

Em todos os perfis de tensões residuais longitudinais, foram visualizadas tensões residuais trativas no lado de avanço, que corresponde ao metal 5083 e, na zona misturada, diminuições nos níveis de tensões residuais, chegando tensões compressivas nos parâmetros 3, 7 e 9. De acordo com Lombard et al. ${ }^{18} \mathrm{o}$ parâmetro dominante para determinar os picos de tensões residuais longitudinais é a taxa de avanço da ferramenta, já que ela determina a entrada de calor por unidade de comprimento da solda. Nesse caso, os parâmetros 3 e 7 apresentam mesma velocidade de avanço ( $35 \mathrm{~mm} / \mathrm{min})$ e de rotação (865 RPM), fornecendo uma entrada de energia mais alta. 
É importante ressaltar que a entrada de energia mais alta leva a um gradiente de temperatura mais acentuado devido a um menor volume de agitação através da ductilidade reduzida do material ao redor da ferramenta, conforme indicado por Colegrove Shercliff \& Zettler ${ }^{19} \mathrm{e}$ isso está relacionado à análise de todos os parâmetros em conjunto. No caso do parâmetro 9 , com velocidade de avanço de $118 \mathrm{~mm} / \mathrm{min}$ e rotação de $642 \mathrm{RPM}$, foi fornecido uma entrada de energia adequada para redução dos níveis de tensões residuais.

Com relação às tensões residuais transversais, todas as juntas soldadas, com exceção das juntas 2, 3 e 7, apresentaram tensões trativas e um perfil constante ao longo da junta soldada. Esses valores obtidos surgem devido à grande dissipação e variação de calor durante a região, apesar de não entrar no estado fundido. Analisando os parâmetros, fica evidente que os menores perfis de tensões residuais trativas são encontrados nos parâmetros 3 e 7 , tanto para as tensões residuais longitudinais como transversais.

Com relação às propriedades mecânicas, foram analisados primeiramente os perfis de dureza das juntas soldadas ao longo da zona misturada (ZM) e do metal de base (MB). Os resultados plotados, observados na Fig. 8, revelam que houve uma invariabilidade nos resultados da dureza no lado de avanço para a zona misturada e um aumento de dureza conforme caminha para o lado de recuo. De acordo com Ahmed et al. ${ }^{20}$ ao soldar juntas dissimilares da liga AA 7075 e AA 5083 observou três regiões distintas no perfil de dureza, sendo a primeira uma região mais macia que corresponde ao lado de avanço com a liga AA 5083, uma

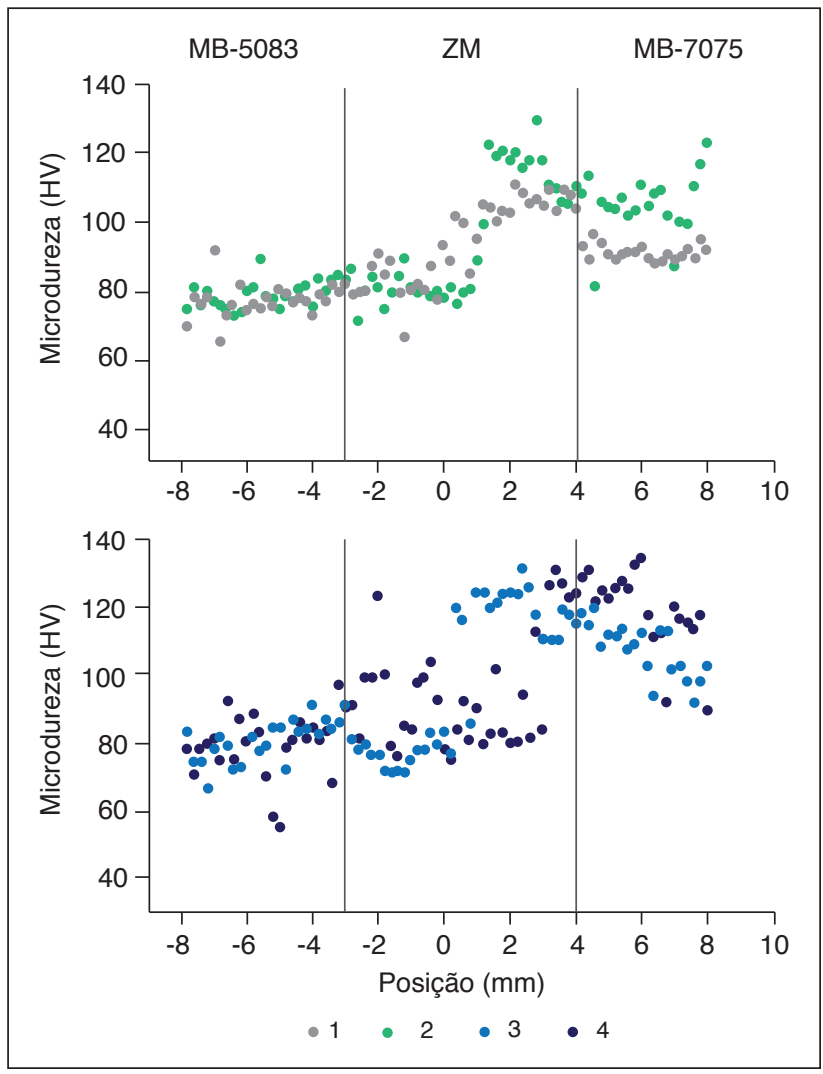

Figura 8: Disposições dos pontos ao longo da junta soldada, onde foi realizado o ensaio de dureza. região de dureza mais elevada, correspondendo ao lado de recuo (AA 7075) e uma região intermediária de dureza, com valores entre as duas zonas distintas, conforme observado na Fig. 8 .

Em ligas tratáveis termicamente, há uma queda na dureza à medida que a temperatura de pico aumenta na região afetada pelo calor. Isso ocorre porque os precipitados irão coalescer e reduzir a densidade numérica em regiões afastadas da fonte de calor, e irá reentrar em solução quando a temperatura máxima for suficientemente alta, de acordo com Sato et al..$^{21}$ como foi observado no lado de recuo, onde se encontra a liga 7075 .

Em geral, quando são realizadas soldagens dissimilares de ligas de alumínio tratáveis termicamente, como no caso AA 6061 e AA 7075, é visualizada certa diminuição da dureza na solda em relação ao seus metais de base correspondentes e isto está associado principalmente ao coalescimento, dissolução e re-precipitação causados pelo ciclo térmico da soldagem pelo processo $\mathrm{FSW}^{21,22}$. Na zona misturada, ocorre re-precipitações após a dissolução completa, visto que o material experimentou uma maior temperatura nessa região ${ }^{1}$

A transição de menores valores de microdureza para maiores valores se torna menos acentuada nos parâmetros 1 e 5, de acordo com a Fig. 9, que ilustra o Boxplot para todos os parâmetros. Esses parâmetros têm em comum tanto a velocidade de avanço (35 mm/min) como a de rotação (345 RPM). Foi observado por Guo et al. ${ }^{1}$, ao realizar soldagens dissimilares entre ligas $6061 \mathrm{e}$ 7075, que menores velocidades de soldagem indicaram uma mistura mais eficaz do material.

A fim de qualificar a ductilidade das juntas soldadas foram realizados ensaios de dobramento de raiz a $60^{\circ}$. Na Tabela 3 é possível observar os valores de carga máxima suportada durante o ensaio para os nove parâmetros. Dentre os parâmetros, a junta 2 apresentou maior valor de carga máxima suportada. De acordo com resultados anteriores, a junta 2 já apresentava um dos menores níveis de tensões residuais trativas. Ao correlacionar a velocidade de avanço de 180 $\mathrm{mm} / \mathrm{min}$, rotação de $345 \mathrm{RPM}$ e sem offset a mistura do material foi eficaz, evitando defeitos que diminuíssem a qualidade da solda.

Já os parâmetros 8 e 4, que possuem em comum a velocidade de avanço $180 \mathrm{~mm} / \mathrm{min}$ e a velocidade de rotação 865 RPM,

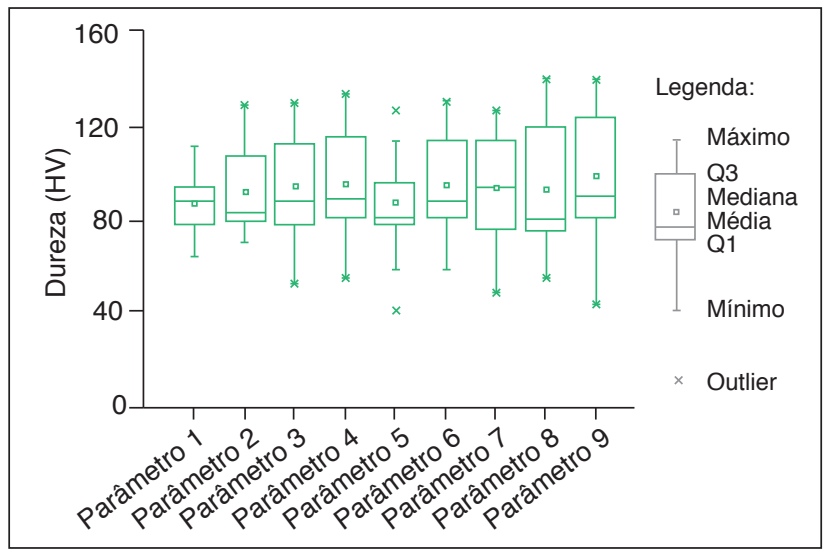

Figura 9: Boxplot dos valores de dureza para os noves parâmetros. 
corresponderam aos menores valores de cargas máximas suportadas. O fato pode ter ocorrido devido aos altos valores de avanço e rotação, que não forneceram tempo adequado para a mistura da junta, fazendo com que ocorra um fluxo inadequado do lado de avanço para o lado de recuo.

Tabela 3: Valores das cargas máximas suportadas no ensaio de dobramento a $60^{\circ}$.

\begin{tabular}{|c|c|c|c|c|}
\hline Juntas & $\begin{array}{c}\text { Carga } \\
\text { máxima } \\
\mathbf{( N )}\end{array}$ & $\begin{array}{c}\text { Desvio } \\
\text { Padrão } \\
\text { da Carga } \\
\mathbf{( N )}\end{array}$ & $\begin{array}{c}\text { Deslocamento } \\
\text { máximo } \\
\mathbf{( m m})\end{array}$ & $\begin{array}{c}\text { Desvio } \\
\text { Padrão do } \\
\text { deslocamento } \\
(\mathbf{m m})\end{array}$ \\
\hline 1 & 2099,117 & 25 & 34,175 & 1,043 \\
\hline 2 & 3012,045 & 43 & 13,637 & 0,875 \\
\hline 3 & 2305,771 & 37 & 29,347 & 0,943 \\
\hline 4 & 1870,221 & 32 & 15,694 & 0,582 \\
\hline 5 & 2421,08 & 35 & 31,339 & 1,102 \\
\hline 6 & 2799,529 & 21 & 21,347 & 0,451 \\
\hline 7 & 2200,013 & 27 & 11,403 & 0,738 \\
\hline 8 & 1766,991 & 30 & 3,857 & 0,073 \\
\hline 9 & 2090,786 & 41 & 24,453 & 0,937 \\
\hline & & & & \\
\hline
\end{tabular}

A coletânea dos ensaios de tração pode ser observada na Fig. 10. De acordo com Ahn et al. ${ }^{23}$, que realizou soldas dissimilares entre ligas da série 6061 com 5083, o comportamento das combinações de materiais diferentes pode ser um pouco diferente um do outro, dependendo da localização do material em relação à direção de deslocamento da ferramenta, que, nesse caso foi encontrado maior valor de limite de resistência à tensão quando a liga não tratável termicamente está no lado de avanço.

Os resultados de combinações de materiais dissimilares mostram que os limites de resistência à tração, são ligeiramente superiores nas combinações dos parâmetros 2 (velocidade de avanço $180 \mathrm{~mm} / \mathrm{min}$ e velocidade de rotação $345 \mathrm{RPM}$ ) e 3 (velocidade de avanço $35 \mathrm{~mm} / \mathrm{min}$ e rotação $865 \mathrm{RPM}$ ). A literatura confirma que maiores velocidades de avanço ocasionam maiores valores de limite de resistência a tração ${ }^{1,21}, o$ que não condiz totalmente com os resultados aqui obtidos, já que os menores valores foram obtidos com velocidades de avanço de $180 \mathrm{~mm} / \mathrm{min}$ e um dos maiores valores obtidos foi com a velocidade de avanço de $35 \mathrm{~mm} / \mathrm{min}$.

Cabe aqui enfatizar a importância da análise dos parâmetros em conjunto. Espera-se que com uma menor entrada de calor ocorram menores gerações de precipitados na zona termicamente afetada ${ }^{1}$. Analisando os resultados, a combinação $35 \mathrm{~mm} / \mathrm{min}$ com rotação 865 RPM e sem offset geram menores aportes de calor na região soldada, apresentando melhores resultados das propriedades mecânicas. Como também é o caso do parâmetro 2, no qual a combinação de velocidade de avanço $180 \mathrm{~mm} / \mathrm{min}$ e velocidade de rotação 345 RPM forneceu melhor mistura ao material, além de melhores propriedades.

Ahmed et al. ${ }^{20}$, ao realizarem soldas dissimilares da liga AA 5083 e AA 7075 verificaram que o limite de resistência obtido nos ensaios variaram entre $245 \mathrm{MPa}$ e 267 $\mathrm{MPa}$, que correspondem a maioria das soldagens realizada pelos diferentes parâmetros, com exceção das juntas 5, 6 e 8, que atingiram valores menores que $200 \mathrm{MPa}$. É importante mencionar que esses valores de limite de resistência à tração são maiores do que o metal de base da liga AA 5083.

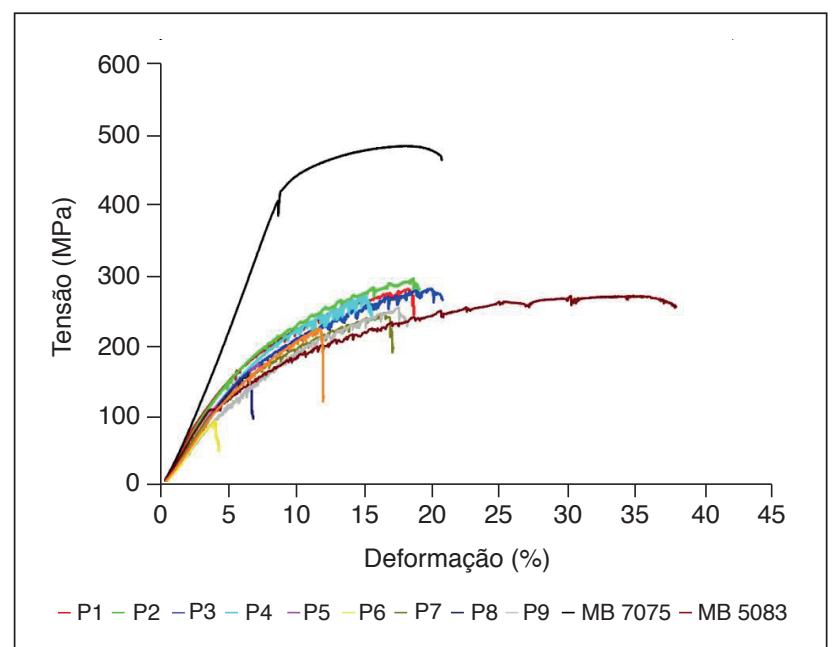

Figura 10: Curva tensão-deformação para as juntas soldadas de alumínio pelo processo FSW e dos Metais de Base.

\section{CONCLUSÕES}

Não foram detectados defeitos superficiais nas juntas soldadas pelos diferentes parâmetros que pudessem comprometer os resultados das propriedades mecânicas. Em relação aos perfis de tensões residuais, foi constatado que a maioria dos parâmetros apresentou valores de tensões residuais longitudinais trativas menores na zona misturada. Já nas tensões residuais transversais, a maioria dos parâmetros se comportou de forma invariável ao longo do perfil. Constatou-se que os parâmetros 2 e 3 forneceram menores valores de tensões residuais trativas entre os demais:

- No perfil de dureza foi visto que houve uma constância da dureza no lado de avanço, para a zona misturada e um aumento de dureza conforme caminha para o lado de recuo, resultados que são comprovados na literatura;

- Comparando os valores obtidos do ensaio de dobramento, foi observado que o parâmetro 2 suportou maiores valores de carga máxima;

- No ensaio de tração o parâmetro 2 apresentou o maior limite de resistência à tração. Esse fato pode estar relacionado à formação de precipitados na zona termicamente afetada, e assim, aumentando os valores de resistência à tração. 


\section{AGRADECIMENTOS}

Os autores agradecem a PRPIPG do Instituto Federal de Educação, Ciência e Tecnologia da Paraíba pelo fomento e bolsa, por meio do edital INTERCONECTA 2018.

\section{REFERÊNCIAS}

1. Guo JF, Chen CN, Sun G, BI G, Sun Z, WeiJ. Friction stir welding of dissimilar materials between AA6061 and AA7075 Al alloys effects of process parameters. Materials and Design. v. 56, p. 185-192. 2014

2. DebroyT.; BhadeshiaH. Friction stir welding of dissimilar alloys a perspective. Science Technol Weld Joining. v. 15, p. 266-270. https://doi.org/10.1179/174329310X12726496072400

3. Murr LE. A review of FSW research on dissimilar metal and alloy systems. J Mater Eng Perform. v. 19, p. 1071-1089. 2010. https:// doi.org/10.1007/s11665-010-9598-0

4. Mishra RS. Friction stir processing technologie s. Advanced Materials \& Process, v. 161, p. 5, 2003.

5. Pao PS, GillSJ,FengCR,SankaranKK. Corrosion - fatigue crack growth in friction stir welded Al 7050. Scripta Materialia, v. 45, p. 605-612, 2001. https://doi.org/10.1016/S1359-6462(01)01070-3

6. LeeY,Jung S. The joint characteristics of Friction Stir Welding MgZnY alloy. Materials Science Forum. v. 475 479, p. 555558. 2005.

7. SuttonMA, Yang B, Reynolds A P, Taylor R. Microstructural studies of friction stir welds in 2024-T3 aluminum. Materials Science and Engineering A, v. 323, p. 160-166, 2002. https://doi.org/10.1016/ S0921-5093(01)01358-2

8. Williams S.W. Welding of Airframes using Friction Stir. AIR \& SPACE EUROPE , v. 3, p. 3, 2001

9. Aval HJ. Microstructure and residual stress distributions in friction stir welding of dissimilar aluminum alloys. Materials and Design. v. 87, p.405-413, 2015. https://doi.org/10.1016/j. matdes.2015.08.050

10. Mishra RS,Ma ZY. Friction stir welding and processing. Materials Science and Engineering: R: Reports, v. 50, n. 1-2, p. 1-78, 8/31/2005.

11. Silva, CC, NetoABS, Silva FDA, Jr FES F, Farias JP. Soldagem de Tubos de Aço ASTM A106 Gr. B para serviço em alta temperatura - Parte 1- Análise das Tensões Residuais. Soldagem \& Inspeção. V. 13 , p. $128-140.2008$.

12. Masubuchi K. Residual stress and distorsion. Metals Handbook. Metals Park, OH, American Society for Metals - ASM. v. 6, 1983.
13. Capelari TV, Mazzaferro JAE. Avaliação da Geometria de Ferramenta e Parâmetros do Processo FSW na Soldagem da Liga de Alumínio AA 5052. Soldagem Insp. São Paulo, Vol. 14, No. 3, p. 215-227, 2009.

14. Souza GS. Influência da Geometria da Ferramenta na Soldagem da Liga de Alumínio 5052 pelo Processo Friction Stir Welding. Dissertação. 2010. 142 p. Dissertação (Mestrado em Engenharia Mecânica). Programa de Pós-Graduação em Engenharia Mecânica e de Materiais, do Campus Curitiba, da UTFPR. Curitiba, Março - 2010.

15. 15. Siqueira FilhoAV. Estudo comparativo das tensões residuais em juntas soldadas pelas técnicas de medição por coordenadas e difração de raios-x. 86 f. Tese (Doutorado) - Universidade Federal de Pernambuco. CTG. Programa de Pós-Graduação em Engenharia Mecânica, Recife, 2012.

16. Richards D, PrangnellP, Withers P, Williams S, Nagy T, Morgan S. Efficacy of active cooling for controlling residual stresses in friction stir welds. Sci. Technol. Weld. Joining. v. 15, p. 156-165. 2010. https://doi.org/10.1179/136217109X12590746472490

17. Altenkirch J, Steuwer A, Peel M, Richards D, Withers P. The effect of tensioning and sectioning on residual stresses in aluminium AA7749 friction stir welds. Mater. Sci. Eng. v. 488, p. 16-24. 2008. https://doi.org/10.1016/j.msea.2007.10.055

18. Lombard H, Hattingh DG, Steuwer A, James MN. Optimising FSW process parameters to minimise defects and maximise fatigue life in 5083-H321 aluminium alloy. Eng Fract Mech. v. 75, p. 341 54. 2008. https://doi.org/10.1016/j.engfracmech.2007.01.026

19. Colegrove PA, Shercliff HR, Zettler R. Model for predicting heat generation and temperature in friction stir welding from the material properties. Sci. Technol.Weld. Joining, v. 12, p. 284-297, 2007. https://doi.org/10.1179/174329307X197539

20. Ahmed MMZ, AtayaS, Seleman MMS, Ammar H R, HamedE. Friction stir welding of similar and dissimilar AA 7075 and AA 5083. Journal of materials processing technology. v. 242, p. 7791. 2017. https://doi.org/10.1016/j.jmatprotec.2016.11.024

21. Sato YS, KokawaH, Enomoto M, Jogan S. Microstructural evolution of 6063 aluminum during friction-stir welding. Metall Mater Trans., v. 30, p 2429 - 37, 1999.

22. Su J Q, Nelson $T$ W, Mishra R, Mahoney M. Microstructura investigation of friction stir welded 7050-T651 aluminium. Acta Mater. v. 51, p.713-729, 2003. https://doi.org/10.1016/S13596454(02)00449-4

23. Ahn EY, Das H, Hong S, Han K, Miles M, Lee K, Park J, Han H. Process responses and resultant joint properties of friction stir welding of dissimilar 5083 and 6061 alumunum alloys. Journal of Mechanical Science and Technology. p. 3955-3960. 2017. 